\title{
Global existence of weak solutions to some micro-macro models
}

\author{
P.-L. Lions ${ }^{\mathrm{a}}$, Nader Masmoudi ${ }^{\mathrm{b}}$ \\ ${ }^{a}$ University Paris-Dauphine, Paris, France \\ ${ }^{\mathrm{b}}$ Courant Institute of Mathematical Sciences 251 Mercer Street New York, NY 10012-1185 USA \\ Received $* * * * * ;$ accepted after revision +++++ \\ Presented by
}

\begin{abstract}
We prove global existence of weak solutions for the co-rotational FENE dumbbell model and the Doi model also called the Rod model. The proof is based on propagation of compactness, namely if we take a sequence of weak solutions which converges weakly and such that the initial data converges strongly then the weak limit is also a solution.

To cite this article: A. Name1, A. Name2, C. R. Acad. Sci. Paris, Ser. I 340 (2005).
\end{abstract}

Key words Fokker-Planck equations, Navier-Stokes equations, FENE model, Doi model, global solutions.

AMS subject classification 35Q30, 82C31, 76A05.

Résumé

Existence globale de solutions faibles a quelques modèles micro-macro

On montre l'existence globale de solutions faibles à certains modèles micro-macro. En particulier on étudie le modèle FENE (le cas des ressorts) et le modèle de Doi (le cas des barres rigides) La preuve est basée sur la propagation de la compacité.

Pour citer cet article: A. Name1, A. Name2, C. R. Acad. Sci. Paris, Ser. I 340 (2005).

\section{Version française abrégée}

\subsection{Le modèle FENE}

On montre l'existence globale de solutions faibles au modèle FENE où les polymères sont considérés comme des ressorts avec une élongation finie. Le systéme est donné par (voir la version anglaise pour les notations).

Email addresses: lions@ens.fr (P.-L. Lions), masmoudi@cims.nyu.edu (Nader Masmoudi). 


$$
\left\{\begin{array}{l}
\partial_{t} u+(u \cdot \nabla) u-\nu \Delta u+\nabla p=\operatorname{div} \tau, \quad \operatorname{div} u=0, \\
\partial_{t} \psi+u \cdot \nabla \psi=\operatorname{div}_{R}\left[-W(u) \cdot R \psi+\frac{\beta}{2} \nabla \psi+\nabla \phi \psi\right] . \\
\tau_{i j}=\int_{B}\left(R_{i} \otimes \nabla_{j} \phi\right) \psi(t, x, R) d R, \quad\left(\nabla \phi \psi+\frac{\beta}{2} \nabla \psi\right) \cdot n=0 \text { on } \partial B\left(0, R_{0}\right) .
\end{array}\right.
$$

Théorème 0.1 Soit $u_{0} \in L^{2}\left(\mathbb{T}^{N}\right)$ et $\psi_{0}$ telle que $\int_{B} \phi_{0} d R=1$ p.p en $x$ et $\int_{B} \frac{\psi^{2}}{\psi_{\infty}} d R \in L_{x}^{\infty}$. Alors, il existe une solution faible globale $(u, \psi)$ de (1) avec $u \in L^{\infty}\left(0, T ; L^{2}\right) \cap L_{\text {loc }}^{2}\left(0, T ; H^{1}\right)$ et $\psi \in L^{\infty}\left(0, T ; L^{\infty}\left(L^{2}\left(\frac{d R}{\psi_{\infty}}\right)\right)\right)$.

0.2. Le modèle de Doi ou modèle rigide

Dans le modèle de Doi, les polymères sont représentés par des barres rigides l'orientation $R$.

$$
\left\{\begin{array}{l}
\partial_{t} u+(u \cdot \nabla) u-\nu \Delta u+\nabla p=\operatorname{div} \tau, \quad \operatorname{div} u=0, \\
\partial_{t} \psi+u . \nabla \psi=\operatorname{div}_{R}\left[-P_{R^{\mid}} \nabla u \cdot R \psi\right]-\Delta_{R} \psi \\
\tau_{i j}=\int_{\mathbb{S}^{N-1}} N\left(R_{i} \otimes R_{j}\right) \psi(t, x, R) d R+b \nabla_{k} u_{l}: \int R_{k} R_{l} R_{i} R_{j} \psi d R,
\end{array}\right.
$$

On démontre le théorème suivant

Théorème 0.2 Soit $u_{0} \in L^{2}\left(\mathbb{T}^{N}\right)$ et $\psi_{0}$ telle que $\int_{\mathbb{S}^{N-1}} \psi_{0} d R=1$ p.p en $x$ et $\int_{\mathbb{T}^{N}} \int_{B} \psi|\log \psi| d R d x<$ $\infty$. On suppose que $b>-\frac{N}{N-1} \nu$. Alors, il existe une solution faible globale $(u, \psi)$ de (2) avec $u \in$ $L^{\infty}\left(0, T ; L^{2}\right) \cap L_{\text {loc }}^{2}\left(0, T ; H^{1}\right)$ et $\left.\psi \in L^{\infty}\left(0, T ; L \log L\left(\mathbb{T}^{N} \times \mathbb{S}^{N-1}\right)\right)\right)$.

\section{Introduction}

Macromolecules play an important role in applied physics, chemistry and biology. Although a polymer molecule may be a very complicated object, there are simple theories to model it. In this note we deal with two models: the FENE model and the Doi model. At the level of the polymeric liquid, we get a system coupling the fluid and the polymers. The Navier-Stokes equation has an extra term due to the polymer force (this is a micro-macro force). Moreover, the Fokker-Planck equation describing the evolution of the polymer density has a drift term which depends on velocity gradient (this is a macro-micro interaction).

\section{The FENE model}

For flexible polymers, a macromolecule is idealized as an "elastic dumbbell" consisting of two "beads" joined by a spring which can be modeled by a vector $R$ (see [2]). The micro-macro approach consists in writing a coupled multi-scale system of the form (1). In $(1), \psi(t, x, R)$ denotes the distribution function for 
the internal configuration and $F(R)=\nabla \phi$ is the spring force which derives from a potential $\phi$. Besides, $\beta$ is related to the temperature of the system and $\nu>0$ is the viscosity of the fluid. $W(u)$ denotes the anti-symmetric part of $\nabla u$, namely $W(u)=\frac{\nabla u-{ }^{t} \nabla u}{2}$. The fact of putting $W(u)$ instead of the whole $\nabla u$ in (1) is done to get better estimate on $\psi$. The system obtained is called the co-rotational FENE model. This is a major simplification of the model.

Here, $R$ can either be in a bounded ball or the whole space $\mathbb{R}^{N}$. If $R$ is in the whole space, the model reduces to the Hooke model for which $\phi(R)=k|R|^{2}$ and the model reduces to the Oldroyd-B model (see [3] and [10] for some local and global existence results). If $R$ is in the ball $B\left(0, R_{0}\right)$, the model reduces to the FENE model (Finite Extensible Nonlinear Elastic) in which case $\phi(R)=-\frac{k}{2} \log \left(1-|R|^{2} /\left|R_{0}\right|^{2}\right)$ for some constant $k>0$ and we have to add a boundary condition to get the conservation of $\psi$, namely $\left(\nabla \phi \psi+\frac{\beta}{2} \nabla \psi\right) \cdot n=0$ on $\partial B\left(0, R_{0}\right)$. The boundary condition on $\partial B\left(0, R_{0}\right)$ insures the conservation of the polymer density and should be understood in the weak sense, namely for any function $g(R) \in C^{1}(B)$, we have

$$
\partial_{t} \int_{B} g \psi d R+u \cdot \nabla_{x} \int_{B} g \psi d R=-\int_{B} \nabla_{R} g\left[-W(u) \cdot R \psi+\frac{\beta}{2} \nabla \psi+\nabla \phi \psi\right] d R .
$$

Notice in particular that it implies that $\psi=0$ on $\partial B\left(0, R_{0}\right)$ and that if initially $\int \psi(t=0, x, R) d R=1$, then for all $t$ and $x$, we have $\int \psi(t, x, R) d R=1$. In the sequel, we only deal with the FENE model and we will take $R_{0}=1$. For simplicity, we assume that $x$ lies in the torus $\mathbb{T}^{N}$ where $N=2$ or $N=3$.

Notice that, the Fokker-Planck equation in (1) can be written as a Stochastic differential equation (see Ottinger [12]). There are many existence results for (1). We can mention Jourdain, Lelievre and Le Bris [8], Weinan E, Li and Zhang [7] and Zhang and Zhang [14], Lin, Liu and Zhang [9] and Barrett, Schwab and Suli [1].

\subsection{A priori estimates and main theorem}

we denote $\psi_{\infty}=e^{\frac{-2 \phi}{\beta}} / \int_{B} e^{\frac{-2 \phi}{\beta}}=\left(1-|R|^{2}\right)^{-k / \beta} / \int_{B}\left(1-|R|^{2}\right)^{-k / \beta}$. For (1), we have the following a priori estimate

$$
\frac{\partial}{\partial t}\left[\int_{\mathbb{T}^{N}} \frac{|u|^{2}}{2}\right]=-\nu \int_{\mathbb{T}^{N}}|\nabla u|^{2}-\int_{\mathbb{T}^{N}} \tau: \nabla u
$$

The second equation of (1) can also be written as

$$
\partial_{t} \psi+u . \nabla \psi=\operatorname{div}_{R}[-W(u) \cdot R \psi]+\operatorname{div}_{R}\left[\psi_{\infty} \nabla \frac{\psi}{\psi_{\infty}}\right]
$$

Hence, for $p>0$, we have

$$
\partial_{t} \int_{B} \psi_{\infty}\left(\frac{\psi}{\psi_{\infty}}\right)^{p} d R+u . \nabla \int_{B} \psi_{\infty}\left(\frac{\psi}{\psi_{\infty}}\right)^{p} d R=-\frac{4(p-1)}{p} \int_{B} \psi_{\infty}\left|\nabla\left(\frac{\psi}{\psi_{\infty}}\right)^{p / 2}\right|^{2} d R .
$$

Notice here, that due to the co-rotational simplification, the term $\operatorname{div}_{R}(-W(u) R \psi)$ has no contribution.

Theorem 2.1 Take $u_{0} \in L^{2}\left(\mathbb{T}^{N}\right)$ and $\psi_{0}$ such that $\int \psi_{0} d R=1$ a.e in $x$ and $\int_{B} \frac{\psi^{2}}{\psi_{\infty}} d R \in L_{x}^{\infty}$. Then, there exists a global weak solution $(u, \psi)$ of (1) with $u \in L^{\infty}\left(0, T ; L^{2}\right) \cap L_{l o c}^{2}\left(0, T ; H^{1}\right)$ and $\psi \in$ $L^{\infty}\left(0, T ; L^{\infty}\left(L^{2}\left(\frac{d R}{\psi_{\infty}}\right)\right)\right)$. 


\section{The Doi model}

Polymers are idealized as rods of fixed length. The configuration space is $R \in \mathbb{S}^{N-1}$. The micro-macro equation is the so-called Doi model (see (2)). In (2), $\Delta_{R}$ is the Laplacian on the sphere and $P_{R}$ is the orthogonal projection on the tangent space to the sphere at $R$, namely $P_{R} \nabla u R=\nabla u R-(R . \nabla u . R) R$ and $b$ is parameter. For the Doi model, we do not need to make the co-rotational simplification since we do not have the potential singularity as in the FENE model. We refer to [13], [4] and [5] for previous results.

\subsection{A priori estimates and main theorem}

The free energy satisfies

$$
\begin{array}{r}
\partial_{t}\left[\int_{\mathbb{T}^{N}} \frac{|u|^{2}}{2}+\int_{\mathbb{T}^{N}} \int_{\mathbb{S}^{N-1}} \psi \log \psi-\psi+1\right] \\
+b \int_{\mathbb{T}^{N}} \nabla_{k} u_{l}: \int_{\mathbb{S}^{N-1}} R_{k} R_{l} R_{i} R_{j} \psi d R: \nabla_{i} u_{j}
\end{array}
$$

To make sure that the free energy is dissipated, we have to assume that $b>-\frac{N}{N-1} \nu$.

Theorem 3.1 Let $u_{0} \in L^{2}\left(\mathbb{T}^{N}\right)$ and $\psi_{0}$ such that $\int_{\mathbb{S}^{N-1}} \phi_{0} d R=1$ a.e in $x$ and $\int_{\mathbb{T}^{N}} \int_{B} \psi \log \frac{\psi}{\psi_{\infty}} d R d x<$ $\infty$. Assume that $b>-\frac{N}{N-1} \nu$. Then, there exists a global weak solution $(u, \psi)$ to (2).

We point out that this theorem also applies to the more general Smoluchowski-Navier-Stokes system studied in [4] and [5].

\section{Idea of the proof of theorem 2.1}

We will only prove the propagation of the compactness of weak solutions to (1). Details of the proofs will be given elsewhere. More precisely take $\left(u^{n}, \psi^{n}\right)$ a sequence of weak solutions to (1) with the initial data $\left(u_{0}^{n}, \psi_{0}^{n}\right)$ which satisfy (4) and (6) and such that $\left(u_{0}^{n}, \psi_{0}^{n}\right)$ converges strongly to $\left(u_{0}, \psi_{0}\right)$ in $L^{2}(d x) \times L^{2}\left(\frac{d R}{\psi_{\infty}} d x\right)$. We extract a subsequence such that $u^{n}$ converges weakly to $u$ in $L^{p}\left((0, T) ; L^{2}\left(\mathbb{T}^{N}\right)\right) \cap$ $L^{2}\left((0, T) ; H^{1}\left(\mathbb{T}^{N}\right)\right)$ and $\psi^{n}$ converges weakly to $\psi$ in $L^{p}\left((0, T) \times \mathbb{T}^{N} ; L^{2}\left(\frac{d R}{\psi_{\infty}}\right)\right)$ for each $p<\infty$. We would like to prove that $(u, \psi)$ is still a solution to (1). We present the proof for $N=2$. The case $N=3$ can be handled using the same modification as in [10]. We also introduce the following defect measures

$$
\begin{aligned}
& \left(\psi^{n}-\psi\right)^{2} \rightarrow \eta, \quad\left|\nabla\left(u^{n}-u\right)\right|^{2} \rightarrow \mu, \quad \psi^{n} \nabla u^{n} \rightarrow \psi \nabla u+\beta \\
& \left|\nabla_{R} \frac{\psi^{n}-\psi}{\psi_{\infty}}\right|^{2} \rightarrow \kappa, \quad\left|\tau^{n}-\tau\right|^{2} \rightarrow \alpha
\end{aligned}
$$

where the convergences are in the sense of distributions. We claim that

$$
\begin{aligned}
\nu \mu & =\int \beta_{i j} R_{i} \nabla_{j} \phi d R \leq C \sqrt{\mu} \sqrt{\alpha}, \quad\left|\beta_{i j}\right| \leq \sqrt{\mu} \sqrt{\eta} \\
\mu & \leq C \alpha \leq C \int\left(\psi_{\infty} \kappa+\frac{\eta}{\psi_{\infty}}\right) d R .
\end{aligned}
$$


The proof of the first assertion is the same as in [10]. The second one is based on Cauchy-Schwarz inequality. The last assertion uses Cauchy-Schwarz inequality and the following Hardy type inequality

$$
\left|\tau^{n}-\tau\right|^{2}=\left|\int\left(\psi^{n}-\psi\right) R_{i} \nabla \phi\right|^{2} \leq C \int \psi_{\infty}\left|\nabla \frac{\left(\psi^{n}-\psi\right)}{\psi_{\infty}}\right|^{2}+\frac{\left(\psi^{n}-\psi\right)^{2}}{\psi_{\infty}} d R
$$

To prove this inequality, we have to distinguish between the cases $\frac{k}{\beta}=1,<1$ or $>1$. We do not detail the proof here (see [11]). On one hand, passing to the limit in the equation for $\psi$, multiplying by $\frac{\psi}{\psi_{\infty}}$ and integrating in $R$, we get

$$
\partial_{t} \int \frac{\psi^{2}}{\psi_{\infty}}+u . \nabla \int \frac{\psi^{2}}{\psi_{\infty}}=\int \operatorname{div}_{R}\left(-R_{j} \frac{\beta_{i j}-\beta_{j i}}{2}\right) \frac{\psi}{\psi_{\infty}}-\int \psi_{\infty}\left|\nabla \frac{\psi}{\psi_{\infty}}\right|^{2}
$$

On the other hand, multiplying (5) by $\frac{\psi^{n}}{\psi_{\infty}}$ and passing to the limit, we get

$$
\partial_{t} \int \frac{\psi^{2}+\eta}{\psi_{\infty}}+u . \nabla \int \frac{\psi^{2}+\eta}{\psi_{\infty}}=-\int \psi_{\infty}\left(\left|\nabla \frac{\psi}{\psi_{\infty}}\right|^{2}+\kappa\right) .
$$

Hence, taking the difference between the two previous equations and using (11), we get

$$
\begin{aligned}
\partial_{t} \int_{B} \frac{\eta}{\psi_{\infty}}+u . \nabla \int_{B} \frac{\eta}{\psi_{\infty}} & \leq C \sqrt{\mu} \int_{B} \sqrt{\eta}\left|\nabla \frac{\psi}{\psi_{\infty}}\right|-\int_{B} \psi_{\infty} \kappa \\
& \leq C \sqrt{\mu}\left(\int_{B} \frac{\eta}{\psi_{\infty}}\right)^{1 / 2}\left(\int_{B} \psi_{\infty}\left|\nabla \frac{\psi}{\psi_{\infty}}\right|^{2}\right)^{1 / 2}-\int_{B} \psi_{\infty} \kappa \\
& \leq C\left(1+\int_{B} \psi_{\infty}\left|\nabla \frac{\psi}{\psi_{\infty}}\right|^{2}\right) \int_{B} \frac{\eta}{\psi_{\infty}}
\end{aligned}
$$

where we have used Cauchy-Schwarz inequality and (12) to control $\mu$ by $\eta$ and $\kappa$. Hence, using that $\int_{B} \frac{\eta}{\psi_{\infty}}=0$ at time 0 , we deduce that $\eta=0$ for all $t$ (see [6] and [10]) and hence $\psi^{n}$ converges strongly to $\psi$ and we can pass to the limit in (5). This proves that the weak limit $(u, \psi)$ is a weak solution of (1).

\section{Idea of the proof of theorem 3.1}

We only prove the propagation of the compactness for a sequence $\left(u^{n}, \psi^{n}\right)$ of weak solutions. For simplicity, we take $b=0$. We assume that $u^{n}$ converges weakly to $u$ in $L^{p}\left((0, T) ; L^{2}\left(\mathbb{T}^{N}\right)\right) \cap L^{2}\left((0, T) ; H^{1}\left(\mathbb{T}^{N}\right)\right)$ and $\psi^{n}$ converges weakly to $\psi$ in $L^{p}\left((0, T) \times \mathbb{T}^{N} ; L^{1}(d R)\right.$ for each $p<\infty$. Let $H=\left(-\Delta_{R}+I\right)^{-s}$ for some $s>N / 2+1$. Hence, $H: L^{1}\left(\mathbb{S}^{N-1}\right) \rightarrow L^{\infty}\left(\mathbb{S}^{N-1}\right)$ and $H \psi^{n}$ is bounded in all variables. We also introduce the following defect measures

$$
\begin{aligned}
\left|H\left(\psi^{n}-\psi\right)\right|^{2} \rightarrow \eta, \quad\left|\nabla\left(u^{n}-u\right)\right|^{2} & \rightarrow \mu, \quad \psi^{n} \nabla u^{n} \rightarrow \psi \nabla u+\beta \\
\left|\nabla_{R} H\left(\psi^{n}-\psi\right)\right|^{2} \rightarrow \kappa, \quad\left|\tau^{n}-\tau\right|^{2} & \rightarrow \alpha
\end{aligned}
$$

Using that

$$
\left|\tau^{n}-\tau\right|=\left|\int N(R \times R)\left(\psi^{n}-\psi\right)\right| \leq C \int H^{-1}(R \times R) H\left(\psi^{n}-\psi\right)
$$


we deduce that $\alpha \leq C \int \eta d R$ is in $L^{\infty}(d t d x)$. Moreover, we have as above $\nu \mu=N \int \beta_{i j} R_{i} R_{j}$ and $\mu \leq$ $C \alpha \leq C \int \eta d R$. Arguing as above, after applying the operator $H$ to the equation of $\psi$, we get

$$
\partial_{t} \int_{S} \eta+u . \nabla \int_{S} \eta=-\int_{S} \overline{H \nabla_{R}\left[-P_{R}\left[\left(\psi^{n} \nabla u^{n}-\psi \nabla u\right) \cdot R\right]\right]\left(H\left(\psi^{n}-\psi\right)\right)}-\int_{S} \kappa
$$

where $\overline{g^{n}}$ denotes the weak limit of $g^{n}$. Using Cauchy-Schwarz, the first term on the right hand side can be estimated by

$$
\leq C\left(\int_{S} \eta\right)^{1 / 2}\left[\mu+|\nabla u|\left(\int_{S} \kappa\right)^{1 / 2}\right] \leq C\left(1+|\nabla u|^{2}\right)\left(\int_{S} \eta\right)+\frac{1}{4} \int_{S} \kappa .
$$

Hence, we deduce that $\partial_{t} \int_{S} \eta+u . \nabla \int_{S} \eta \leq C\left(1+|\nabla u|^{2}\right) \int_{S} \eta$ and since, $\int_{S} \eta=0$ at time 0 , we deduce that $\eta=0$ for all $t$ and hence $(u, \psi)$ is a weak solution of $(2)$.

\section{Acknowledgments}

The work of N. M. is partially supported by NSF-DMS grant 0403983 .

\section{References}

[1] J. W. Barrett, C. Schwab, and E. Süli. Existence of global weak solutions for some polymeric flow models. Math. Models Methods Appl. Sci., 15(6):939-983, 2005.

[2] R. B. Bird, C. Curtiss, R. Amstrong, and O. Hassager. Dynamics of polymeric liquids, Kinetic Theory Vol. 2,. Wiley, New York, 1987.

[3] J.-Y. Chemin and N. Masmoudi. About lifespan of regular solutions of equations related to viscoelastic fluids. SIAM J. Math. Anal., 33(1):84-112 (electronic), 2001.

[4] P. Constantin, C. Fefferman, E. Titi, and A. Zarnescu. Regularity for coupled two-dimensional nonlinear fokker-planck and navier-stokes systems. Preprint, 2006.

[5] P. Constantin and N. Masmoudi. Global well posedness for a Smoluchowski equation coupled with Navier-Stokes equations in 2d. preprint, 2007.

[6] R. J. DiPerna and P.-L. Lions. Ordinary differential equations, transport theory and Sobolev spaces. Invent. Math., 98(3):511-547, 1989.

[7] W. E, T. Li, and P. Zhang. Well-posedness for the dumbbell model of polymeric fluids. Comm. Math. Phys., 248(2):409$427,2004$.

[8] B. Jourdain, T. Lelièvre, and C. Le Bris. Existence of solution for a micro-macro model of polymeric fluid: the FENE model. J. Funct. Anal., 209(1):162-193, 2004.

[9] F.-H. Lin, C. Liu, and P. Zhang. On hydrodynamics of viscoelastic fluids. Comm. Pure Appl. Math., 58(11):1437-1471, 2005.

[10] P.-L. Lions and N. Masmoudi. Global solutions for some Oldroyd models of non-Newtonian flows. Chinese Ann. Math. Ser. B, 21(2):131-146, 2000.

[11] N. Masmoudi. Well posedness for the FENE dumbbell model of polymeric flows. Preprint, 2007.

[12] H. C. Öttinger. Stochastic processes in polymeric fluids. Springer-Verlag, Berlin, 1996. Tools and examples for developing simulation algorithms.

[13] F. Otto and A. Tzavaras. Continuity of velocity gradients in suspensions of rod-like molecules. Preprint, 2005.

[14] H. Zhang and P. Zhang. Local existence for the FENE-dumbbell model of polymeric fluids. Arch. Ration. Mech. Anal., 181(2):373-400, 2006. 